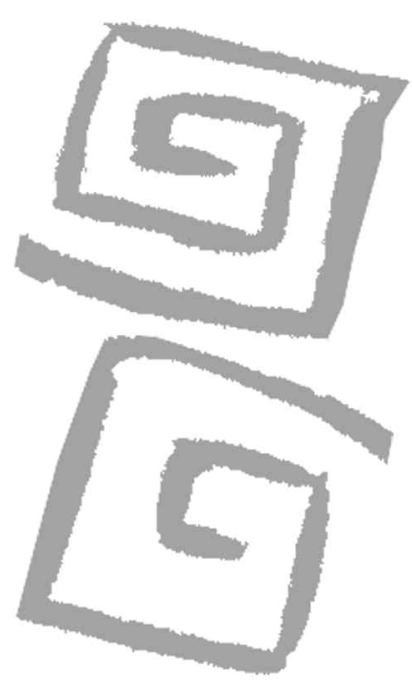

${ }^{1}$ Licenciado en Sociología. Investigador, Intercambios Asociación Civil. Investigador, Instituto Gino Germani, Universidad de Buenos Aires, Argentina.

gonzaloralon@yahoo.com.ar

${ }^{2}$ Trabajadora Social. Especialista en Problemáticas Sociales Infanto-juveniles. Coordinadora, Área de Investigación, Intercambios Asociación Civil. Buenos Aires, Argentina. drossi@intercambios.org.ar

${ }^{3}$ Médico. Especialista en Clínica Médica y Epidemiología. Asesor médico e investigador, Intercambios Asociación Civil. Buenos Aires, Argentina. marceevila@gmail.com

${ }^{4}$ Licenciada en Sociología. Instituto de Investigación y Desarrollo Social (IDES). Montevideo, Uruguay. llatorre2@gmail.com

${ }^{5}$ Médico. Doctor en Salud Pública. Investigador, Fundação Oswaldo Cruz. Río de Janeiro, Brasil.bastos@cict.fiocruz.br

${ }^{6}$ Médica. Doctora en Ciencias. Directora del Observatório de Saúde Urbana \& Grupo de Pesquisas em Epidemiologia, Faculdade de Medicina, Universidade Federal de Minas Gerais. Belo Horizonte, Brasil. wcaiaffa@medicina.ufmg.br

\section{De los estudios locales a una perspectiva regional: análisis integrado de datos secundarios en un proyecto colaborativo sobre vulnerabilidades asociadas al uso de drogas en Argentina, Brasil y Uruguay (1998-2004)}

\author{
From local studies to a regional perspective: pooled \\ analysis of secondary data in a collaborative project on \\ vulnerabilities associated with drug use in Argentina, \\ Brazil and Uruguay (1998-2004)
}

Ralón, Gonzalo ${ }^{1}$; Rossi, Diana2; Vila, Marcelo3; Latorre, Laura4; Bastos, Francisco Inácio ${ }^{5}$; Caiaffa, Waleska Teixeira ${ }^{6}$

RESUMEN Este artículo desarrolla los fundamentos metodológicos del diseño de análisis integrado a partir de su empleo para el estudio de situaciones de vulnerabilidad entre usuarios de drogas a nivel regional. Se integraron datos de trece estudios transversales realizados en Argentina, Brasil y Uruguay entre 1998 y 2004. Una revisión crítica del concepto de matriz de datos, que distingue cuatro componentes estructurales, permitió: definir las unidades de análisis abarcando las distintas poblaciones originales; identificar un núcleo común de variables (características sociodemográficas, uso de drogas, prácticas sexuales, serologías de infecciones de transmisión sexual y sanguínea) con sus respectivos valores; examinar los indicadores, las dimensiones y procedimientos empleados para la medición de las variables; y establecer su compatibilidad mediante un análisis comparativo y temático de los instrumentos. Se obtuvo una nueva matriz de 3.534 casos. La colaboración multidisciplinaria entre equipos e instituciones de los tres países hizo posible maximizar las fuentes disponibles para el análisis de características locales y de la región en su conjunto.

PALABRAS CLAVE Metodología; Análisis de Datos; Consumidores de Drogas; VIH; Transmisión de Enfermedad Infecciosa.

\begin{abstract}
This paper develops the methodological principles of pooled analysis design, using it to study situations of vulnerability among drug users at a regional level. Data from thirteen cross-sectional studies carried out in Argentina, Brazil and Uruguay between 1998 and 2004 were integrated. A critical review of the concept of data matrix which identifies four structural components, allowed us to: define the units of analysis spanning the different original populations; identify a core of common variables (social and demographic characteristics, drug use, sexual practices, serology of blood-borne and sexually transmitted diseases) with their respective values; examine the indicators, dimensions and procedures used to measure the variables; and establish their compatibility with a thematic and comparative analysis of data collection tools. The main result was a new data matrix with 3,534 cases. Multidisciplinary collaboration between teams and institutions from the three countries made it possible to maximize the available sources in order to analyze characteristics of the local contexts and of the overall regional.
\end{abstract}

KEY WORDS Methodology; Data Analysis; Drug Users; HIV; Infectious Disease Transmission. 


\section{INTRODUCCIÓN}

Aunque los datos recientes muestran que la transmisión del virus de la inmunodeficiencia humana $(\mathrm{VIH})$ tiende a estabilizarse en varios países de América Latina y el Caribe (1), la epidemia sigue concentrándose en poblaciones con situaciones de alta vulnerabilidad. Entre ellas, los usuarios de drogas $y$, en particular, aquellos que se inyectan drogas y comparten materiales para la inyección aparecen como los grupos con mayores prevalencias junto con los hombres que tienen sexo con hombres, las personas transgénero, los trabajadores sexuales, las personas privadas de libertad (2-4). La vulnerabilidad frente al VIH y otras infecciones se extiende también a las personas que conforman las redes sexuales y de consumo de drogas de esos grupos.

Argentina, Brasil y Uruguay han sido históricamente los países sudamericanos en los que el uso inyectable de drogas tuvo mayor extensión $(5,6)$, en especial en los grandes centros urbanos de la cuenca del Plata -Buenos Aires, Montevideo, Rosario y sus áreas periféricas-, del sur y sudeste de Brasil -Porto Alegre, Florianópolis y São Paulo-, como también en Salvador, en el norte. Desde fines de los años ochenta, los casos de sida registrados en los tres países evidenciaron que el uso compartido de materiales de inyección tendría un gran peso en la epidemia y, aunque la transmisión por el uso compartido de materiales de inyección viene reduciéndose desde mediados de los años noventa (7), esta reducción fue acompañada por referencias cada vez más frecuentes a la transmisión de VIH y de otras infecciones de transmisión sexual y sanguínea (ITSS) entre usuarios de drogas no inyectables (8-14). Ambos grupos, además de ser vulnerables a la infección de VIH y la coinfección con virus de la hepatitis B y C (VHB y $\mathrm{VHC})$, suelen tener un acceso limitado y tardío a la atención sanitaria, con una alta proporción de diagnósticos en etapas sintomáticas $(15,16)$.

Todo esto ubica a los usuarios de drogas en el centro de una problemática socio-sanitaria que desborda los ámbitos locales. Su dimensión regional hace necesario desarrollar e implementar diseños de investigación que habiliten análisis más complejos, ampliando el alcance temporal y territorial de los datos. En este artículo se discute el modelo de análisis integrado -pooled analysis o integrative data analysis en inglés- que tuvo una primera formalización en el trabajo de Christine Friedenreich (17-20). Se trata de un tipo de diseño destinado a sintetizar evidencias empíricas obtenidas por distintas fuentes que, a diferencia de los diseños de revisión sistemática o metaanálisis basados en resultados publicados, integra datos en bruto que permiten nuevos análisis. Se describe aquí su empleo con datos de trece estudios epidemiológicos, transversales y descriptivos, realizados entre 1998 y 2004 con usuarios de drogas de Argentina, Brasil y Uruguay. Los fundamentos del proceso de integración se revisan desde una perspectiva crítica que difiere substancialmente de las concepciones metodológicas predominantes en epidemiología, y que se basa en las reflexiones del epistemólogo y metodólogo argentino Juan Samaja sobre la estructura del dato científico (21) (a).

\section{LA ESTRUCTURA DEL DATO CIENTÍFICO}

...donde el dato fetichizado era, la desconstrucción [sic] y la reelaboración deben devenir. Juan Samaja (21 p.390).

Trabajar con datos secundarios -producidos en función de problemas, hipótesis y objetivos que no son los de la presente investigaciónrequiere tomar, en principio, al dato en sí mismo como objeto. Pero no puede entenderse al dato como manifestación directa e inmediata de un objeto y de sus atributos. La transformación de la experiencia sensible en objeto científico ofrece como resultado datos, unidades elementales de información que posibilitan la reelaboración de esas experiencias recortando la percepción inmediata, empobreciendo su riqueza bruta, su multiplicidad, su heterogeneidad, pero enriqueciéndola al concentrar la percepción en aspectos de la realidad relevantes en el marco de los modelos teóricos del investigador. No se trata de entidades predefinidas que puedan ser simplemente recolectadas en el campo. Los datos nunca están dados. Son, por el contrario, construcciones complejas que articulan un contenido empírico, referido a un estado de cosas en el mundo al que se accede sensorialmente, con otro de tipo teórico, conceptual. 
Una perspectiva clásica, presente en buena parte de la literatura metodológica de distintas disciplinas, concibe a la matriz de datos como una estructura de tres partes $(22,23)$ : unidades de análisis, variables y valores. Su función se limita a la comparación entre unidades de análisis, posibilitando el cálculo de medidas de resumen, de tendencia central, de dispersión. Esta concepción obstaculiza la comprensión de la génesis del dato, de su historia formativa, perdida en el misterio de una funcionalidad consagrada y cerrada: la matriz en calidad de herramienta de análisis de datos no da cuenta de cómo se obtienen los valores en cada caso (b).

Samaja (21) propone una revisión de esta perspectiva que sintetiza en tres postulados:

1. Los datos de cualquier investigación científica, cualquiera sea la disciplina que se trate, poseen una misma estructura invariante, general, universal, que llamamos matriz de datos.

2. Esta estructura consta no de tres, sino de cuatro elementos: las unidades de análisis, las variables y los valores identificados tradicionalmente, a los que se agregan los indicadores como parte de la estructura del dato.

3. En toda investigación los datos describen al objeto de estudio en diferentes niveles analíticos interrelacionados conformando un sistema de matrices. En su forma más simple, el sistema puede pensarse en tres niveles: de anclaje, nivel de las unidades de análisis centrales en la investigación; subunitario, cuyas unidades son partes componentes, aspectos particulares de las unidades del nivel de anclaje que permiten describirlas; y nivel supraunitario, correspondiente a los contextos en que las unidades del nivel de anclaje están insertas y que determinan en alguna medida sus características. Los datos concebidos como sistemas de matrices destacan la multiplicidad de niveles lógicos y ontológicos del objeto de estudio en toda su complejidad.

En este punto hay que destacar las implicancias teórico-metodológicas de definir al indicador como un componente estructural del dato antes que como un tipo especial de variable que por sí misma permite caracterizar un fenómeno determinado (tal la definición corriente de indicador en metodología de la investigación, en epidemiología y en otras ciencias). Desde una perspectiva crítica, toda variable, en la medida en que requiere de una definición de los criterios de aprehensión empírica, de medición, implica un indicador que vincula el contenido conceptual de la variable con un estado de cosas del mundo empírico por medio de una praxis específica. El indicador es también una estructura compleja: son los procedimientos u operaciones que, aplicados a una dimensión, aspecto o manifestación parcial y observable de la variable, aprehensible por medio de los sentidos, permiten determinar qué valor de esa variable le corresponde a cada unidad de análisis, cómo clasificarla en función de esa variable. Así, este concepto de indicador devela el misterio del proceso de construcción de la evidencia empírica.

Con estas definiciones presentes, la integración de los datos exige su deconstrucción (c): penetrar en su estructura y alcanzar analíticamente sus componentes elementales para contrastar las definiciones conceptuales y operacionales en las que se apoyan. Solo así puede identificarse qué rasgos de qué entidades fueron observados y registrados, y mediante qué procedimientos.

A continuación se describe la integración de sistemas de matrices de diferentes estudios (Cuadro 1), con los cuatro componentes estructurales del dato como ejes de comparación. Así se pudo, en primer lugar, elaborar una definición común de las unidades de análisis, abarcadora de las poblaciones originales con un nivel de generalidad mayor. El análisis de protocolos, informes de investigación, documentos de gestión y publicaciones en los que se explicitaban los criterios de inclusión originales fueron la fuente para esta comparación. Luego se identificó un núcleo de variables comunes a los trece estudios, sus sistemas de valores y sus respectivos indicadores. Comparar los indicadores permitió establecer la identidad cualitativa de las características estudiadas en cada una de las investigaciones. En este caso, la fuente de comparación residió en el análisis temático de los cuestionarios y en la contrastación de los paquetes serológicos empleados en cada estudio, que permitió hacer explícitas las definiciones operacionales y las operacionalizaciones. 
Cuadro 1. Períodos, localidades, poblaciones, serologías y tamaños muestrales de los estudios originales. 1998-2004.

\begin{tabular}{|c|c|c|c|c|c|c|c|c|c|}
\hline \multirow[t]{2}{*}{$\mathbf{N}^{\circ}$} & \multirow[t]{2}{*}{ Período } & \multirow[t]{2}{*}{ Localidades } & \multirow[t]{2}{*}{ Población } & \multicolumn{5}{|c|}{ Serologías } & \multirow{2}{*}{$\begin{array}{l}\text { Tamaño } \\
\text { muestral }\end{array}$} \\
\hline & & & & VIH & VHC & VHB & TP & HTLV I-II & \\
\hline 1 & 1998 & $\begin{array}{l}\text { Porto Alegre (RS) } \\
\text { Itajaí (SC) } \\
\text { São José do Rio Preto (SP) } \\
\text { São Paulo - Apta (SP) } \\
\text { Sorocaba (SP) }\end{array}$ & UDI & Sí & Sí & No & No & Sí & 287 \\
\hline 2 & 1998 & $\begin{array}{l}\text { Ciudad Autónoma de Buenos Aires } \\
\text { Gran Buenos Aires }\end{array}$ & UDI & No & No & No & No & No & 60 \\
\hline 3 & $1999-2001$ & Río de Janeiro & UDI, ex UDI, no UDI & Sí & Sí & Sí & Sí & No & 632 \\
\hline 4 & 1999-2002 & Ciudad Autónoma de Buenos Aires & UD (pasta base) & No & No & No & No & No & 25 \\
\hline 5 & $2000-2001$ & Gran Buenos Aires & UDI, ex UDI & $\mathrm{Si}^{\prime *}$ & Sí & Sí & No & Sí & 174 \\
\hline 6 & $2000-2002$ & $\begin{array}{l}\text { Salvador de Bahia (BA) } \\
\text { São José do Rio Preto (SP) } \\
\text { Florianópolis (SC) } \\
\text { Itajaí (SC) } \\
\text { Porto Alegre (RS) } \\
\text { Gravataí (RS) }\end{array}$ & UDI & Sí & Sí & Sí & Sí & Sí & 821 \\
\hline 7 & $2002-2003$ & $\begin{array}{l}\text { Ciudad Autónoma de Buenos Aires } \\
\text { Gran Buenos Aires }\end{array}$ & UD (cocaína inhalada) & No & No & No & No & No & 150 \\
\hline 8 & $2002-2003$ & $\begin{array}{l}\text { Ciudad Autónoma de Buenos Aires } \\
\text { Gran Buenos Aires }\end{array}$ & UD (cocaína no inyectada) & $\mathrm{Si}^{*}$ & Sí & Sí & Sí & No & 502 \\
\hline 9 & $2002-2003$ & $\begin{array}{l}\text { Montevideo } \\
\text { Gran Montevideo }\end{array}$ & UD (cocaína no inyectada) & $\mathrm{Si}^{\prime *}$ & Sí & Sí & Sí & No & 367 \\
\hline 10 & 2003 & Ciudad Autónoma de Buenos Aires & UDI & No & No & No & No & No & 99 \\
\hline 11 & 2003 & $\begin{array}{l}\text { Montevideo } \\
\text { Gran Montevideo }\end{array}$ & UDI, ex UDI & $\mathrm{Si}^{\prime *}$ & Sí & Sí & No & No & 200 \\
\hline 12 & 2003-2004 & $\begin{array}{l}\text { Ciudad Autónoma de Buenos Aires } \\
\text { Gran Buenos Aires }\end{array}$ & UDI & No & No & No & No & No & 154 \\
\hline 13 & 2004 & Gran Buenos Aires (Avellaneda) & UDI & No & No & No & No & No & 63 \\
\hline
\end{tabular}

Fuente: Elaboración propia a partir de datos de los estudios originales 1 (26,27); 2 (28); 3 (29,30); 4 (31); 5 (32); 6 (26,33); 7 (34,35); 8 (13); 9 (10); 10 (36); 11 (37); $12(36,38) ; 13(39)$.

*Los casos con serología de VIH positiva fueron analizados con el test de seroconversión reciente (STARHS) para la estimación de incidencia anualizada. $\mathrm{VIH}=$ Virus de la inmunodeficiencia humana. $\mathrm{VHC}=$ Virus de la hepatitis C. VHB = Virus de la hepatitis B. TP = Treponema pallidum. HTLV I-II = Human Tlymphotropic virus type I-II (virus linfotrópico humano de células T tipo I-III). UDI = Usuarios de drogas inyectables. UD = Usuarios de drogas,

\section{UNIDADES DE ANÁLISIS}

Las poblaciones centrales de los estudios originales fueron diversos grupos de usuarios de drogas. En un nivel subordinado, los proyectos se concentraron en las prácticas de uso de drogas y prácticas sexuales y, a nivel contextual, en las condiciones de vida determinadas por los marcos jurídicos, institucionales y políticos. Las unidades de observación y de información, en todos los estudios coincidían con las unidades de análisis del nivel central: los usuarios de drogas.

Tres estudios trabajaron con otras poblaciones que conformaban lo que Samaja
(21) denomina matrices de datos coordinadas, que no fueron incluidos en la matriz integrada. Se trataba de unidades de análisis independientes aunque del mismo nivel de integración que las del nivel de anclaje y que constituían grupos de comparación y control: las parejas sexuales de los usuarios de drogas $(34,35)$ o jóvenes sin experiencia reciente de uso inyectable (39), usaran o no drogas por otras vías.

A su vez, cada población fue originalmente definida con criterios específicos. Comparar los criterios de inclusión permitió definir a la población integrada como una síntesis de las definiciones conceptuales y operativas originales, abarcando grupos con diversas prácticas. 


\section{Criterios de inclusión}

- Territorialidad: Dos estudios implementaron diseños multicéntricos en un total de ocho ciudades de Brasil. Las otras poblaciones fueron contactadas en Buenos Aires, Montevideo y Río de Janeiro y en las localidades periféricas que conforman sus respectivas áreas metropolitanas (Cuadro 2). Esto no implicó que todos los participantes residieran en esas ciudades. Pero la selección por técnicas intencionales, de bola de nieve, o aun coincidentales en los escenarios de uso de drogas permitió acceder a los usuarios de drogas pertenecientes o muy vinculados a las poblaciones locales.

- Lugares de contacto: En un estudio de Buenos Aires y otro de Montevideo $(10,13)$ se incluyeron usuarios de drogas institucionalizados, internados en centros para el tratamiento de problemáticas relacionadas con el uso de drogas, tanto públicos como privados.

- Edad: Dos estudios (29-31) admitieron la participación de usuarios de drogas con 15 o 16 años, y un tercer proyecto (28) no especificó una edad mínima. En el resto se excluyeron los menores de 18 años.

- Sexo: En todos los estudios participaron varones y mujeres; en buena parte de ellos se establecieron cuotas por sexo para asegurar un mínimo de información sobre mujeres usuarias de drogas.

- Uso de drogas: Las definiciones originales fueron diversas pero se orientaron hacia poblaciones de usuarios de drogas con alta vulnerabilidad epidemiológica y social, definidas con dos criterios superpuestos. Primero, por las substancias consumidas: drogas ilegales y legales utilizadas sin prescripción médica. No obstante, la ilegalidad de las drogas, o su uso irregular o no prescripto en el caso de las legales, no bastaba para definir grupos vulnerables en términos de sus prácticas. El segundo criterio apuntó a las vías de consumo. Nueve proyectos trabajaron con usuarios de drogas inyectables. La dimensión temporal del uso inyectable tuvo distintas definiciones: mientras algunos estudios abarcaban a quienes se hubieran inyectado drogas alguna vez en la vida, sin especificar frecuencia ni condiciones de uso inyectable, otros consideraban un plazo largo (alguna vez en los últimos 10 o 20 años), un período reciente o actual; actualidad que, a su vez, comprendía desde los 3 hasta los 12 meses previos a la encuesta. Los estudios con usuarios de drogas no inyectables se concentraron sobre todo en consumidores de cocaína, crack y/o pasta base de cocaína. Dos de esos estudios $(10,13)$ excluyeron a los usuarios de drogas inyectables, concentrándose en quienes no tenían experiencia de uso inyectable. El resto admitió la participación de usuarios de drogas inyectables siempre que cumplieran con los criterios mínimos relativos al uso no inyectable.

- Condición serológica: Las serologías estaban destinadas a estimar prevalencias, de modo que no se excluyó a quienes ya conocían su condición serológica positiva.

- Participación voluntaria e información: Criterio compartido por todos los proyectos que aseguraban la confidencialidad y anonimato de las informaciones brindadas. Todos los participantes otorgaron su consentimiento por escrito. En los estudios con serologías se informó a los participantes sobre el alcance de las pruebas y se les brindó soporte para afrontar los resultados y acceder a tratamientos.

Cuadro 2. Distribución porcentual de la muestra integrada por país y localidad. 1998-2004.

\begin{tabular}{lcc}
\hline País y localidad & \multicolumn{2}{c}{ Muestra } \\
\cline { 2 - 3 } & $\mathbf{n}$ & $\%$ \\
\hline Argentina & & \\
$\quad$ Buenos Aires & 1.227 & 34,7 \\
Uruguay & & \\
$\quad$ Montevideo & 567 & 16,0 \\
Brasil & & \\
$\quad$ Río de Janeiro & 632 & 17,9 \\
$\quad$ Porto Alegre & 390 & 11,0 \\
$\quad$ Salvador & 194 & 5,5 \\
$\quad$ São José do Rio Preto & 167 & 4,7 \\
$\quad$ Itajaí & 140 & 4,0 \\
$\quad$ Gravataí & 111 & 3,1 \\
$\quad$ Florianópolis & 52 & 1,5 \\
$\quad$ Sorocaba & 38 & 1,1 \\
$\quad$ São Paulo & 16 & 0,5 \\
Total & 3.534 & 100,0 \\
$\quad$ & \\
\hline Fuente: Elaboración propia a partir de datos integrados (10,13,26-39). & \\
&
\end{tabular}


Figura 1. Comparación de instrumentos (codificación de preguntas y campos de registro y contrastación de algoritmos y paquetes serológicos) y procesamiento de datos.

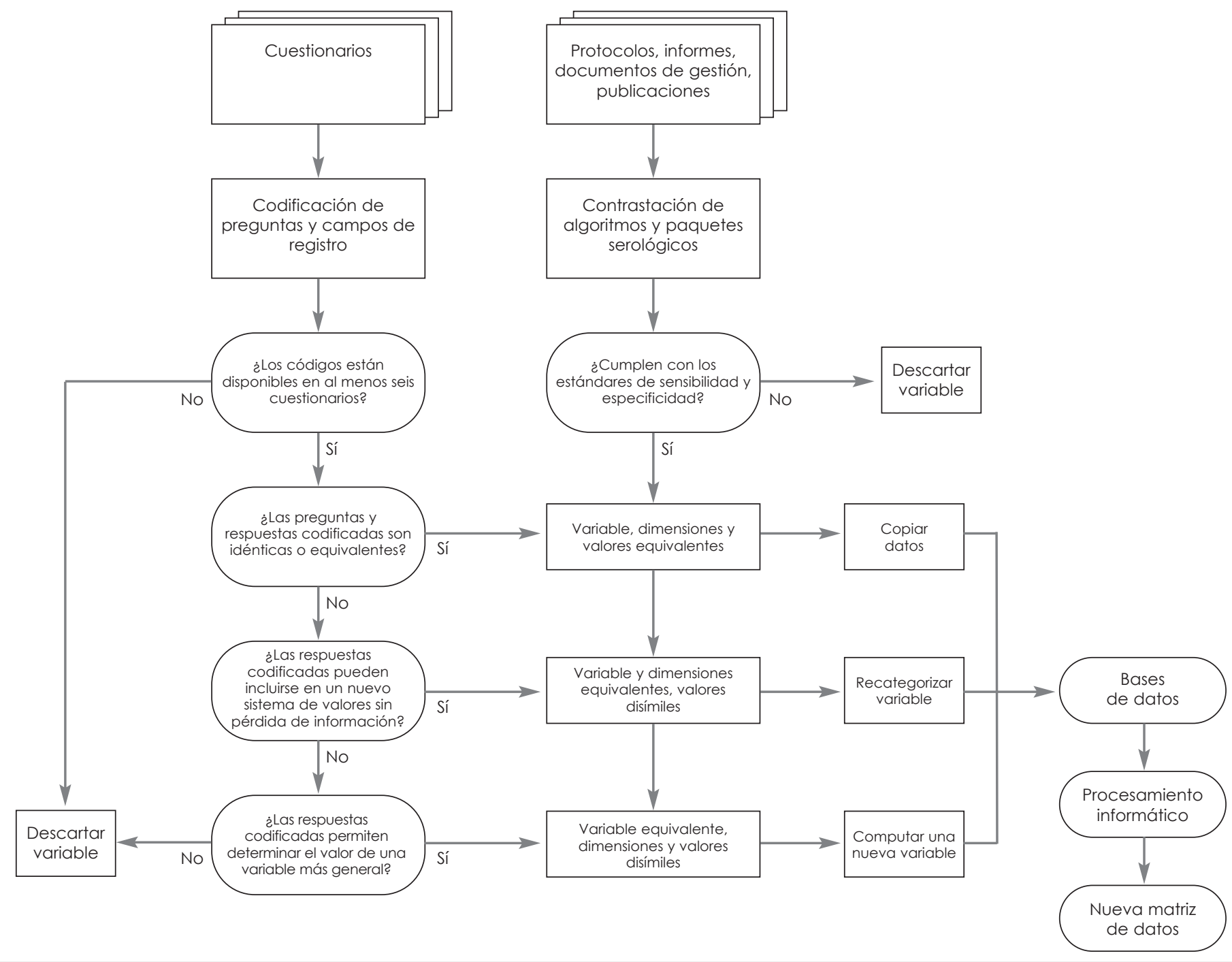




\section{Acceso a las poblaciones}

Los trece proyectos originales emplearon diseños muestrales no probabilísticos, intencionales, con selección coincidental o por bola de nieve. Aunque estos diseños conllevaban la importante limitación de no permitir la inferencia de parámetros con probabilidades conocidas ni el control de ciertos sesgos sistemáticos aleatorizando la selección constituían los mejores métodos disponibles para acceder a poblaciones de difícil acceso, no institucionalizadas, ocultas.

El contacto inicial con muchos de los usuarios de drogas fue posible por el trabajo de intervención y asistencia realizado en cada contexto por diferentes organizaciones sociales y por instituciones que participaron o colaboraron con los estudios. Algunos fueron contactados y encuestados en la Policlínica de Farmacodependencia del Hospital Maciel y el Hospital de Referencia Psiquiátrica Vilardebó, de Montevideo (10), y el Centro Nacional de Reeducación Social de Buenos Aires (13).

\section{VARIABLES, VALORES E INDICADORES}

\section{Instrumentos}

El análisis de los instrumentos se fundamenta por su estrecha relación con los indicadores. Los instrumentos condensan en un dispositivo material dos instancias esenciales en la construcción del dato (21): las definiciones operacionales, es decir, la selección de dimensiones conceptuales relevantes y aprehensibles empíricamente; y la operacionalización, el diseño de los procedimientos concretos que deben aplicarse en cada caso para aprehender el contenido conceptual de la variable.

Se siguieron dos caminos según los tipos de instrumentos: el análisis temático de los trece cuestionarios utilizados en los estudios originales, asistido por el software Atlas.ti versión 5.5, y la contrastación de algoritmos y paquetes serológicos (Figura 1) .

El análisis temático es un método comúnmente utilizado en investigaciones cualitativas para identificar, analizar y describir patrones dentro de un corpus discursivo textual o no textual $(40,41)$. Permite organizar y describir con cierto grado de detalle los contenidos y avanzar en la interpretación de diversos aspectos del tema de investigación. En este caso, el propio texto de los cuestionarios fue el corpus de análisis. En todos los estudios se usaron cuestionarios semiestructurados con campos de registro y preguntas cerradas, semiabiertas y en menor medida, abiertas. El análisis temático buscó identificar las dimensiones relevantes y los procedimientos prescriptos, plasmados en los instrumentos.

Por otra parte, se compararon los algoritmos serológicos. Se utilizaron paquetes comerciales de enzimoinmunoanálisis (ELISA por sus siglas en inglés Enzyme-linked immunosorbent assay) para la detección de VIH, VHB, VHC y del virus linfotrópico humano de células T tipo I-II (HTLV III por sus siglas en inglés Human T-lymphotropic virus type $I-I I)$ combinados en algunos casos con métodos de cribado por aglutinación de partículas; y también VDRL (por sus siglas en inglés Venereal disease research laboratory) para detectar sífilis. Todas las pruebas cumplían con los estándares de sensibilidad y especificidad para estimaciones de prevalencia válidas y confiables (d).

Además, algunas variables se obtuvieron mediante dimensiones no registradas con los instrumentos originales, rasgos derivados del contexto en que se realizó el trabajo de campo: país, ciudad, período de tiempo, estudio e identificadores de casos. Esos rasgos permitieron crear variables contextuales, emergentes del nivel supraunitario, características de los contextos a los que pertenecían las unidades del nivel de anclaje. Sus indicadores surgen del análisis de un conjunto de metadatos que superan el contenido estricto de las matrices originales.

\section{Cuestionarios, preguntas, respuestas}

El análisis temático de los cuestionarios siguió tres pasos, similares a los señalados por Brandt et al. para la integración de cuestionarios de investigaciones clínicas (43). En primer lugar, la codificación de preguntas y campos de registro en función de su temática. Se identificaron nueve ejes temáticos relevantes: identificación de unidades de análisis en contextos, características sociodemográficas, encarcelamiento, acceso a 
servicios de salud y tratamiento por drogas, formas de uso de drogas, substancias consumidas, uso compartido de materiales de inyección, prácticas sexuales, diagnósticos previos de VIH, ITSS y tuberculosis.

Luego, la selección de variables relevantes disponibles. Para asegurar una mínima masa de datos sobre cada uno de los conceptos de interés se seleccionaron aquellos códigos que referían a campos de registro relevantes por su contenido, disponibles en al menos seis de los estudios originales.

Una vez identificadas las variables relevantes, el análisis de los cuestionarios permitió comparar las formulaciones de cada pregunta -es decir, cómo se preguntó a los encuestados-y, en función de la temática de cada pregunta, identificar las dimensiones de las variables. Con esas dimensiones presentes, se compararon los indicadores y se evaluó su compatibilidad.

\section{Resultados del análisis temático-comparativo de los cuestionarios}

Cuatro fueron los resultados posibles:

1. Las variables podían integrarse directamente pues las preguntas y los conjuntos de respuestas de cada cuestionario, si bien no eran idénticos en su formulación, eran de significado equivalente. Es decir que, aunque los procedimientos podían diferir en sus formas, se aplicaban a las mismas dimensiones de variables equivalentes, con sistemas de categorías también equivalentes. En estos casos hubo que considerar la cuestión lingüística, las diferencias entre el castellano de Buenos Aires, el de Montevideo, y el portugués de las urbes brasileñas. Pero también, los diferentes significados que tenían el potencial de denotar y connotar los términos usados en cada contexto. Como en toda encuesta, la operacionalización requirió desarrollar transposiciones semánticas en dos direcciones: de los conceptos a los términos nativos para hacer referencia a las prácticas, a sus condiciones concretas y a sus efectos objetivos y subjetivos en un lenguaje comprensible para los encuestados; y luego, de los términos nativos de las respuesta, a los contenidos conceptuales. Interesó para este análisis advertir qué términos se utilizaron en cada cuestionario para referirse a los fenómenos que se buscaba describir. Esto se manifestaba en la redacción de las preguntas, en los usos literales y/o figurativos de ciertos términos para referir a algunas nociones, y en la relación entre denominaciones comunes, médico-científicas, comerciales y jurídicolegales de las substancias, de las prácticas y de las condiciones en que se desarrollaban. Así, la identificación de dimensiones equivalentes requirió comparar los usos particulares del lenguaje en cada contexto.

2. Las variables podían integrarse estableciéndose un nuevo sistema de categorías (respuestas) capaz de abarcar los valores originales sin una pérdida importante de información. Para ello fue indispensable recategorizar variables basadas en dimensiones equivalentes, pero compatibilizando sistemas de categorías disímiles, con diferentes niveles de medición -nominal, ordinal, de intervalo, de razón-, como la frecuencia de uso inyectable durante el último lapso de consumo o el momento en que se usaron drogas inyectadas por última vez. En estos casos, los valores debían referir a períodos de tiempo compatibles y, a la vez, conceptualmente significativos. Otro caso de este tipo es el de las variables sobre educación, cuyos valores originales respondían a los sistemas educativos argentino, brasileño y uruguayo. Las diferencias entre países y entre provincias en Argentina se solventaron agrupando los años de escolaridad formal en dos categorías ordinales de mayor nivel de generalidad, pero que maximizaban la cantidad de respuestas disponibles: hasta siete años -educación primaria completa en el sistema argentino clásico- y más de siete años de educación.

3. Variables con dimensiones diferentes podían integrarse en una variable de mayor nivel de generalidad. En estos casos, las dimensiones de las variables y sus valores eran disímiles, pero existían preguntas cuyas respuestas atañían a aspectos equivalentes del objeto. Por ejemplo, la pregunta por la cantidad de hijos, en contraste con la pregunta acerca de si tenían o no hijos. La segunda solo admitía una categorización nominal entre los que tenían y los 
que no tenían; la primera, en cambio, permitía obtener una escala de números naturales: $0,1,2 \ldots \mathrm{n}$ hijos. Al integrarlas, sus valores se sintetizaron en dos variables de diferentes niveles de medición: una variable dicotómica que permitió disponer de una mayor cantidad de respuestas con lo valores sin hijos, por una parte, y con uno o más hijos, por la otra; y otra variable de nivel de medición intervalar, referida a la cantidad de hijos. Del mismo modo, preguntas sobre frecuencias de uso inyectable, edad de inicio en el uso inyectable, uso compartido de materiales, substancias inyectadas alguna vez, aunque con dimensiones más específicas, permitían determinar si el encuestado se había inyectado drogas alguna vez en la vida.

Otra variable de este tipo surgía de combinar distintas preguntas sobre uso de preservativo o, en términos más generales, sobre prácticas de cuidado en las relaciones sexuales (e). Las dificultades en este caso fueron grandes porque las preguntas originales referían a aspectos diferentes del uso del preservativo según: tipo de pareja (estable u ocasional, del mismo o del otro sexo), tipo de penetración, situación (en la última relación, en las más recientes), proporción o frecuencia con que se usó, y distintos períodos de tiempo. A su vez, diferían entre valores dicotómicos (sí/no) y ordinales (siempre/a veces/nunca o siempre/la mayoría de las veces/a veces/casi nunca/nunca). Para integrarlas se construyó un índice con todas las dimensiones disponibles, destinado a identificar si los sujetos daban cuenta de no-usar preservativo en algún caso, en cualquiera de las condiciones, situaciones y tipos de prácticas referidas. Se obtuvo así una aproximación a la consistencia general de uso de preservativo por parte de los usuarios de drogas.

4. Ciertas variables carecían de indicadores compatibles en las matrices originales. Una buena proporción de las preguntas originales no presentaba equivalencias, reduciéndose la cantidad de datos disponibles porque los criterios de medición, es decir, las dimensiones seleccionadas y los valores eran substancialmente disímiles.

\section{RECONSTRUCCIÓN}

Algunas de las dimensiones identificadas en los instrumentos sirvieron para construir variables de respuestas múltiples basadas en grupos de preguntas dicotómicas - personas con las que se convivía, acceso a servicios de salud o compañías más frecuentes en el consumo de drogas- o preguntas abiertas -principales fuentes de ingreso, con hasta tres respuestas simultáneas y diferentes entre dieciséis posibles- que admitían más de una respuesta.

La teoría de Samaja permite advertir, en este punto, el carácter polifuncional de algunos indicadores: la observación de un aspecto particular y concreto del objeto permite, en muchos casos, determinar el valor que le corresponde a la unidad de análisis en más de una variable. En otros casos, dimensiones diferentes también permiten determinar el valor de una misma variable. Por ejemplo, la edad, cuyas dimensiones originales eran tanto los años cumplidos como la diferencia en años enteros entre la fecha de encuesta y la fecha de nacimiento. Ambas dimensiones cumplían con los requisitos de validez por cuanto hacían referencia a aspectos relevantes y observables.

Los procedimientos, a su vez, aseguraban un mínimo de confiabilidad: se puede asumir que las variaciones en los valores no estaban sesgadas sino que respondían fundamentalmente a los diferentes estados del objeto, aunque los resultados también podían variar por diferencias de rigurosidad y exactitud en la ejecución de los procedimientos. Aun así, los indicadores resultaron confiables pues posibilitaban el control de sesgos sistemáticos.

Este concepto de indicador también da cuenta de la complejidad de los datos, como constructos que conjugan, mediante prácticas específicas, un contenido teórico con otro de tipo empírico. El empleo de índices es otra muestra cabal de ello. Siguiendo la perspectiva de Samaja, el índice es un tipo de indicador en el que los procedimientos -observación, medición, interrogación, etc.se aplican, no sobre una, sino sobre varias dimensiones de la misma variable que, por relaciones lógicas y/o aritméticas, permiten obtener como resultado un valor determinado. Por ejemplo, para conocer el uso compartido de materiales de 
inyección alguna vez en la vida se consideró el haber compartido al menos una vez: agujas, jeringas, recipientes para mezclar substancias, filtros, líquidos de limpieza de agujas y jeringas o cualquier otro material cuyo uso compartido posibilitara la transmisión de enfermedades. Hasta cinco dimensiones conjugadas por relaciones lógicas -en este caso, que se hubiera compartido uno o más de esos materiales al menos una vez en la vida- conforman un índice complejo para la misma variable.

Así, la deconstrucción de los datos permitió alcanzar sus componentes estructurales, identificar dimensiones y procedimientos en un movimiento eminentemente analítico. Restaurar su estructura y avanzar hacia una reconstrucción requirió un movimiento de sentido inverso, sintético, para pasar de las estructuras abstractas a los datos concretos, integrados en un nuevo sistema de matrices. La integración material se realizó mediante rutinas informáticas de procesamiento de bases de datos. Estas rutinas permitieron identificar algunas inconsistencias en las bases. Se consultaron los registros originales y las notas de campo para comprobar si se trataba de errores de procesamiento y/o carga de las encuestas, o errores en su aplicación. La corrección de estos errores no muestrales, derivados del procesamiento original, permitió mejorar la calidad de los datos integrados.

\section{La muestra integrada}

Alrededor de los diseños de revisión sistemática y metaanálisis se han realizado críticas poniendo en duda la validez de integrar y analizar resultados obtenidos por distintas fuentes (45-47). En su estupendo artículo, a 25 años de la presentación pionera del moderno diseño de metaanálisis (48), Gene Glass reflexiona sobre ciertas críticas que recibiera su propuesta, una de las cuales sostenía que el metaanálisis "mixed apples and oranges" (49). La respuesta del investigador norteamericano se destaca por una profundidad epistemológica no tan frecuente en la literatura metodológica: comparar objetos diversos -como naranjas y manzanas- que conforman una categoría más amplia, para advertir sus diferencias y semejanzas, es parte fundamental del quehacer científico.

En el caso de este análisis integrado, cada una de las poblaciones fue definida originalmente asignándole ciertas características constantes específicas, expresadas en los criterios de inclusión y relativas a sus prácticas de uso de drogas. La integración subsumió esas características dentro de una categoría más amplia, de mayor nivel de generalidad.

Lo que en la metáfora de Glass correspondería a las frutas, en la que se incluyen tanto manzanas como naranjas, es aquí la categoría de los usuarios de drogas. Mezclar objetos diversos -en este caso, usuarios de drogas inyectables con usuarios de drogas no inyectables- como si fueran naranjas y manzanas, adquiere sentido pues permite comparar grupos con distintas prácticas. Así, muchas de las que eran constantes en las poblaciones originales -ser usuario de drogas inyectables o no inyectables, ser de Argentina, de Brasil o de Uruguay según el proyecto- funcionan ahora como variables que permiten identificar diferentes subgrupos en la muestra integrada.

La integración apuntó fundamentalmente al uso de drogas ilegales: heroína, cocaína y derivados de cocaína y/o de su proceso de producción, como la pasta base de cocaína o el crack. También se incluyeron algunas drogas legales: anfetaminas y benzodiacepinas utilizadas sin prescripción médica regular. La diversidad de substancias responde al perfil de policonsumo de estos grupos. La mitad de los participantes había utilizado tres o más tipos de drogas a lo largo de su vida, con un máximo de diez, incluyendo drogas ilegales, alcohol y medicamentos no prescriptos.

Se consideró tanto el uso inyectado como no inyectado. Entre los participantes que nunca se habían inyectado drogas, los consumos incluían: drogas fumadas (básicamente crack, pasta base, cocaína y heroína), inhaladas (como la cocaína) y algunas drogas consumidas por vía oral (benzodiacepinas, ácido lisérgico y otros alucinógenos, codeína, anfetaminas, metanfetaminas, metilendioximetanfetamina -éxtasis- y otras drogas sintéticas). 


\section{Una nueva matriz}

De los 3.605 registros disponibles, 71 correspondían a menores de 18 años o a casos con incongruencias entre distintos indicadores de edad. Se excluyeron esos casos obteniéndose una muestra integrada de 3.534 usuarios de drogas encuestados a lo largo de 7 años, entre el 6 de marzo de 1998 y el 28 de octubre de 2004.

La matriz integrada contiene 79 variables, cuatro de respuestas múltiples que combinan entre 3 y 10 dimensiones. En cuanto a los niveles de medición, cinco variables son de razón, discretas, referidas a cantidades: años de edad, años de educación formal aprobados, edad de inicio en el consumo de drogas y en el consumo de drogas inyectadas, y cantidad de hijos. También se cuenta con las fechas de nacimiento, de encuesta, y el año de realización de la última prueba de VIH. Otras tres variables son ordinales: nivel educativo, período en que se inyectaron drogas por última vez y frecuencia de uso inyectado. El resto de las variables son nominales, en su mayoría dicotómicas, por sí o por no.

No todas las variables de la matriz integrada tienen el mismo tamaño muestral, lo que hubiese requerido un diseño de análisis integrado prospectivo (17) en el que todos los estudios compartieran la misma matriz, similares definiciones operacionales e instrumentos comunes. Las cantidades de respuestas válidas en cada variable están dadas por los datos originales: como se señaló antes, no todos los estudios disponían de las mismas variables, y muchos de los datos disponibles no eran compatibles. Aun así, la variable simple con la menor cantidad de respuestas registra 992 valores efectivos, superando por 171 casos a la muestra original más grande.

De la matriz integrada, 724 casos corresponden a mujeres $(20,5 \%)$ y 2.808 a varones $(79,5 \%)$. En dos casos no se obtuvieron valores para la variable sexo. El 70\% (2.474) corresponde a poblaciones de usuarios actuales o ex usuarios de drogas inyectables y el resto a usuarios de drogas no inyectables (1.060).

Además, la matriz integrada tiene datos sobre grupos con diferentes grados de proximidad y posibilidades de acceso a los servicios de salud, en su mayor parte no institucionalizados: 3.436 casos -el $97,2 \%$ de la muestra integrada- fueron
Cuadro 3. Resultados positivos a pruebas serológicas de VIH, VHB, VHC, sífilis y HTLV I-II correspondientes a los estudios originales integrados. 1998-2004.

\begin{tabular}{lcccc}
\hline \multirow{2}{*}{$\begin{array}{l}\text { Tipo de prueba } \\
\text { serológica }\end{array}$} & $\begin{array}{c}\text { Pruebas } \\
\text { serológicas } \\
\text { realizadas }\end{array}$ & \multicolumn{2}{c}{$\begin{array}{c}\text { Resultados } \\
\text { positivos }\end{array}$} \\
\cline { 3 - 5 } & & $\mathbf{n}$ & $\%$ \\
\hline & & & \\
VIH (ELISA) & 2.976 & 687 & 23,1 \\
Virus Hepatitis C (ELISA) & 2.843 & 914 & 32,1 \\
Virus Hepatitis B (ELISA) & & & \\
Anticuerpo del core & 1.813 & 914 & 32,1 \\
Antígeno de superficie & 2.653 & 567 & 16,0 \\
Sífilis (VDRL) & 1.672 & 567 & 16,0 \\
HTLV I-II (ELISA) & 1.259 & 632 & 17,9 \\
& & &
\end{tabular}

Fuente: Elaboración propia a partir de datos integrados (10,13,26-39).

$\mathrm{VIH}=$ Virus de la inmunodeficiencia humana. $\mathrm{VHB}=$ Virus de la hepatitis $\mathrm{B}$. $\mathrm{VHC}=$ Virus de la hepatitis C. HTLV I-II = Human T-lymphotropic virus type I-II (virus linfotrópico humano de células T tipo I-II). ELISA = Enzyme-linked immunosorbent assay (ensayo por inmunoabsorción ligado a enzimas). VDRL $=$ Venereal disease research laboratory.

contactados en ámbitos y situaciones propios de los usuarios de drogas. Solo 98 casos, de dos estudios de Buenos Aires y Montevideo, fueron encuestados en centros en los que estaban internados para tratarse por problemas relativos al uso de drogas.

El Cuadro 3 presenta las serologías disponibles en cada uno de los estudios originales, con un máximo de 2.976 resultados para $\mathrm{VIH}$ y un mínimo de 1.259 para HTLV I-II $(84,2 \%$ y $35,6 \%$ de la muestra integrada), las prevalencias obtenidas con los datos integrados y los tamaños muestrales de cada serología. Cuando se analizan las prevalencias de cada una de las infecciones -a modo de variable de efecto- por sexo, solo la prevalencia de hepatitis C está asociada a la variable sexo, siendo mayor entre los varones $(34,7 \%)$ que entre las mujeres $(22,7 \%)$.

Por último, la Figura 2 presenta las prevalencias de cada infección según uso inyectable de drogas alguna vez. Con la única excepción de las pruebas de anticuerpos para treponema pallidum y considerando que en los estudios con población de usuarios de drogas no inyectables no se realizaron serologías de HTLV I-II, el resto de las prevalencias son superiores entre los usuarios de drogas inyectables. Pero las altas prevalencias de $\mathrm{VIH}, \mathrm{VHC}$ y $\mathrm{VHB}$ entre los usuarios de drogas no inyectables, varias veces superiores a las estimadas en población general de los tres 
Figura 2. Prevalencias de VIH, VHB, VHC, TP y HTLV I-II según uso inyectable de drogas alguna vez, correspondientes a los estudios originales integrados. 1998-2004.

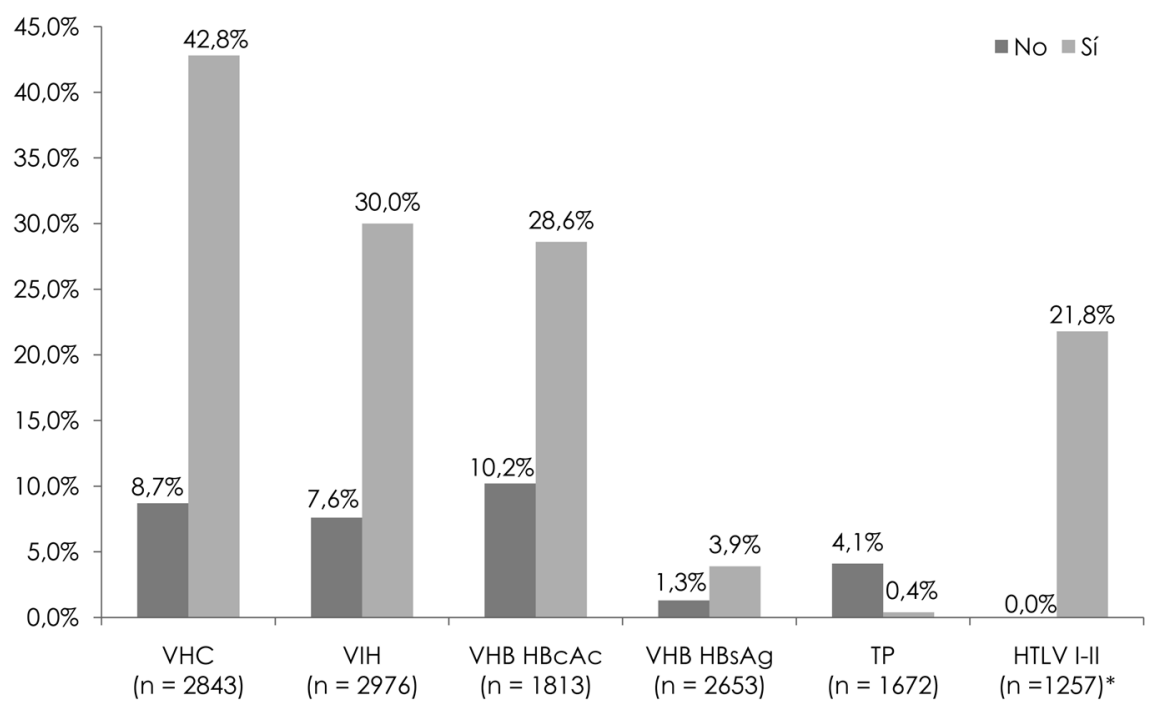

Fuente: Elaboración propia a partir de datos integrados $(10,13,26-39)$.

*En los estudios con usuarios de drogas inyectables no se realizaron serologías de HTLV I-II.

$\mathrm{VIH}=$ Virus de la inmunodeficiencia humana. VHB = Virus de la hepatitis B. VHC = Virus de la hepatitis C. TP = Treponema pallidum. HTLV I$\|=$ Human T-lymphotropic virus type I-II (virus linfotrópico humano de células T tipo I-II). HBCAC = Anticuerpo del core. HBsAg = Antígeno de superficie.

países (1,50-53) coincide con la tendencia identificada en el estado del arte (8-14), y señala la importancia de ampliar la perspectiva de análisis para considerar las situaciones de vulnerabilidad en un sentido amplio, incluyendo otras prácticas que hacen posible la transmisión de ITSS.

\section{DISCUSIÓN}

El análisis integrado es un tipo heterodoxo de diseño de investigación con datos secundarios, poco utilizado en los medios locales (f). Además, no ha sido muy frecuente el uso de este tipo de diseño para la integración de datos epidemiológicos que permitieran caracterizar a las poblaciones en términos sociodemográficos y de tipos de prácticas.

Este proyecto, entonces, es original en muchos aspectos. Su propósito es habilitar una serie de análisis amplios que permitan comparar datos de distintos contextos y resultados parciales de cada estudio, con los que surgen de los datos integrados. Pero además, disponer de una matriz integrada a partir de los datos en bruto, más que de los resultados parciales de cada estudio original -como en un diseño de metaanálisis- permite trabajar con esos datos en función de otros problemas, otras hipótesis y otros objetivos nuevos y propios, y eludir y superar el sesgo de publicación de los resultados originales (17).

Asimismo, se incrementan las posibilidades de análisis por efecto del tamaño muestral $(n=3.534)$, inédito en investigaciones locales con usuarios de drogas, y por la calidad de los datos, mejorada por la detección y corrección de errores presentes en las matrices originales. Ese tamaño muestral permite identificar y conocer mejor las situaciones de vulnerabilidad de grupos muy reducidos en las muestras originales. Por ejemplo, entre los usuarios de drogas: las mujeres y en particular aquellas que tienen relaciones sexuales con mujeres -población que en la 
muestra integrada tiene 94 casos, mientras que en ninguno de los estudios originales superaba los 25 casos-; los varones que tienen relaciones con varones -627 casos en la muestra integrada contra un promedio de 52,2 por estudio y un máximo de 209 en los estudios originales-; aqueIlos con diferentes situaciones laborales, con acceso a distintos tipos de servicios de salud -públicos, privados, laborales-, con experiencias previas de encarcelamiento o de tratamiento por problemas relacionados con el uso de drogas.

Muchos de los estudios originales fueron las primeras investigaciones extensivas de tipo epidemiológico desarrolladas con usuarios de drogas en los tres países. Esto implicó que algunos diseños tuvieran un carácter exploratorio o descriptivo que redundó en una mayor heterogeneidad de los datos disponibles. Además, el carácter no probabilístico de las muestras impidió conocer con qué probabilidad podían ser representativas de cada población. La muestra integrada arrastra estas limitaciones originales. Por lo tanto, las conclusiones derivadas del análisis integrado deberán considerarse como hipótesis sobre tendencias generales en grupos específicos de usuarios de drogas.

Sin embargo, estas investigaciones, también por su carácter inaugural en muchos casos, se desarrollaron a partir de problemas, hipótesis y objetivos similares, orientados a conocer mejor las condiciones de vida, las prácticas de consumo y sexuales, y la condición serológica de distintos grupos de usuarios de drogas, para permitir un diagnóstico de situación en relación con las epidemias de VIH e ITSS; coincidencias que posibilitaron la integración, permitiendo maximizar el conocimiento sobre el tema, identificar problemáticas y situaciones comunes entre usuarios de drogas de los tres países en el período 19982004, e identificar -una vez más, a modo de hipótesis- posibles tendencias con sus proyecciones actuales, a partir de las fuentes disponibles.

Con respecto a la integración de los datos hay que señalar que es un proceso complejo que se desarrolla en un doble sentido: desde la deconstrucción de los datos originales y de su estructura, la abstracción conceptual de las variables, hasta el nivel concreto, elemental, relativo a sus dimensiones empíricas y, a partir de allí, reemprendiendo el camino de la síntesis, desde la redefinición de las poblaciones y de las variables, hasta la integración y consolidación de una nueva matriz de datos. Las tesis de Juan Samaja sobre la estructura del dato científico han sido el fundamento conceptual del proceso. Los análisis sucesivos darán cuenta de las potencialidades y de la validez de la matriz integrada.

En cuanto a su desarrollo práctico, por su complejidad -y también por su singularidadel proceso de integración requirió mucho tiempo y mucho trabajo. Como han señalado Blettner et al. en su tipología de diseños de revisión e integración de datos secundarios (17) y Brandt et al. en su propuesta para la comparación de cuestionarios (43), el trabajo coordinado entre los equipos de investigación, productores primarios de los datos, es indispensable para este tipo de diseño. El acceso a los datos, su deconstrucción, la interpretación, comparación y selección de los indicadores, su reestructuración y la integración final, solo fueron posibles por la comunicación y colaboración estrecha entre investigadores de los tres países y de las instituciones de cada país.

El uso de diseños e instrumentos comunes -como las encuestas de comportamiento en Consumidores de Drogas de Alto Riesgo (CODAR), desarrolladas por investigadores de diferentes países de América y de Europa, que buscan constituir un instrumento estandarizado (55), y que han empezado a utilizarse en investigaciones con usuarios de drogas de nuestra región (7)- facilitará en lo sucesivo el desarrollo de análisis integrados prospectivos.

Para ello es fundamental continuar trabajando con una agenda de investigación común, consensuada, en la que pueda conjugarse el tratamiento de las problemáticas comunes a la región junto con los intereses específicos de cada grupo en cada contexto local. Esa agenda debe prever la integración de nuevos datos, producidos más recientemente, en otros ámbitos locales y nacionales, y con otras subpoblaciones.

En la medida en que el proceso de integración no se cierre y la masa de datos pueda incrementarse, las posibilidades de análisis se ampliarán con la solidez de los resultados. Y, en este sentido, el acceso amplio a la información pública y al acervo de la producción científica, así como el soporte oficial a este tipo de proyectos, han sido y seguirán siendo fundamentales para posibilitar la cooperación entre los distintos equipos de todos los países. 


\section{NOTAS FINALES}

a. Juan Samaja (1941-2007). Sociólogo de la Universidad de Buenos Aires, diplomado en Salud Pública. Docente y organizador de varias carreras de grado y posgrado en diversas universidades latinoamericanas; titular de la cátedra Metodología de la Investigación en Psicología, Facultad de Psicología, Universidad de Buenos Aires. Doctor en Ciencias por la Escuela Nacional de Salud Pública Sérgio Arouca de la Fundação Oswaldo Cruz, con la tesis Fundamentos epistemológicos de las ciencias de la salud (1997), dirigida por Maria Cecília de Souza Minayo.

b. De la perspectiva formalizada por el politólogo noruego Johan Galtung (22) y adoptada, entre otros, por Paul Lazarsfeld (23), se desprende que esa estructura de tres partes corresponde a los datos en ciencias sociales -Galtung no se pronuncia sobre su validez en otras disciplinas-, y que cada investigación involucra tan solo una matriz. Samaja comenta: "Tanto la presuposición de que en una investigación es posible hablar de una sola matriz de datos, como la de que es una estructura tripartita, en donde no figuran los indicadores -ambos supuestos- tienen la gran desventaja de ocultar las numerosas transiciones, mediaciones y transformaciones que ocurren durante la creación científica" (21 p.169).

c. El concepto deconstrucción, que remite al pensamiento del filósofo francés Jáques Derrida, Ileva a interpretar las prácticas científicas como textualidades en sentido amplio, como prácticas discursivas y metadiscursivas (24). Señala Esther Díaz: "Deconstruir la herramienta indispensable del investigador, el lenguaje, forma parte de una propedéutica de los métodos y puede colaborar a la explicitación de los supuestos y la flexibilización de los abordajes, dos instancias exigidas por el rigor científico cuando es alentado por el espíritu crítico, pero descuidadas en la práctica científica cuando se rige por una obediencia acrítica a los mandatos establecidos" (25 p.268).

d. Una descripción de los métodos serológicos empleados para la estimación de la incidencia del VIH en estudios transversales realizados en Brasil, y el análisis de sus ventajas y limitaciones puede encontrarse en el trabajo de Morgado y Bastos (42).

e. Martin et al. (44) presentan un interesante análisis integrado destinado a evaluar la efectividad del uso de preservativo para prevenir la transmisión del virus del herpes simple tipo II a partir de seis estudios diferentes, basados en problemas, objetivos y tipos de diseños distintos, y da cuenta de la integración de datos con diferentes sistemas de categorías referidos al uso de preservativo a partir de procedimientos y dimensiones diferentes.

f. Durante la revisión de este artículo llegó a nosotros el trabajo de Francisco José Dutra Souto et al., publicado en el número de mayo de 2012 del Journal of Medical Virology (54), en el que se presenta un análisis integrado de tipo epidemiológico, realizado con datos de nueve estudios de corte transversal realizados entre 1993 y 2008 en el estado de Mato Grosso, Brasil. Ese análisis integrado se dirige a ampliar las estimaciones de prevalencia de VHC y a evaluar las asociaciones de riesgo en distintos grupos, con una muestra integrada de 3.910 casos. Es la primera referencia conocida a un trabajo de este tipo en la región.

\section{AGRADECIMIENTOS}

Este trabajo fue desarrollado en el marco de los proyectos: "Perfis e tendências de comportamentos de riscos para a infecção pelo HIV/AIDS e Hepatites B e C em Usuários de Drogas no contexto das políticas publicas vigentes: analise entre paises da América Latina", Programa Sul-Americano de Apoio a Cooperação em Ciência e Tecnologia (PROSUL), financiado por el Conselho Nacional de Desenvolvimento Científico e Tecnológico (Edital No 014 - 490426/2006-9) de Brasil; y “Políticas públicas de control de drogas en América Latina" (UBACyT 20020100101021), Instituto de Investigaciones Gino Germani, Facultad de Ciencias Sociales, Universidad de Buenos Aires. Fondos adicionales fueron proporcionados por Fogarty International Center/AIDS International Training and Research Program at Mount Sinai School of Medicine - Argentina Program (NIH Grant \# D43 TW001037). Agradecemos especialmente la colaboración de los doctores Samuel R. Friedman (National Development and 
Research Institutes, New York) y David Vlahov (University of California School of Nursing, San Francisco), que impulsaron esta investigación y de la magíster Marie-Cécile Bertard (Université Paris $\mathrm{V}$ René Descartes, Université Laval, Québec) que colaboró con el procesamiento informático de los datos. Se agradece también a los revisores por sus comentarios, observaciones y sugerencias.

\section{REFERENCIAS BIBLIOGRÁFICAS}

1. ONUSIDA. Global report: UNAIDS report on the global AIDS epidemic 2010 [Internet]. 2010 [citado 10 may 2012]. Ginebra: Joint United Nations Programme on HIV/AIDS; 2010. Disponible en: http://www.unaids.org/globalreport/Global_report.htm.

2. Malta M, Magnanini M, Mello M, Pascom AR, Linhares $\mathrm{Y}$, Bastos F. HIV prevalence among female sex workers, drug users and men who have sex with men in Brazil: A systematic review and meta-analysis. BMC Public Health. 2010;10(1):317.

3. Pando MA. Aportes a la epidemiología del HIV en Argentina: Prevalencia e Incidencia en grupos vulnerables. Actualizaciones en Sida. 2010;18(68):1-8.

4. ONUSIDA/MSPRO. Informe nacional sobre los progresos realizados en la aplicación del UNGASS: Uruguay, 2010. Montevideo: ONUSIDA, Ministerio de Salud Pública, Programa Prioritario ITS/SIDA; 2010.

5. Aceijas C, Friedman SR, Cooper HLF, Wiessing L, Stimson GV, Hickman M. Estimates of injecting drug users at the national and local level in developing and transitional countries, and gender and age distribution. Sexually Transmitted Infections. 2006;82(suppl 3):S10-S17.

6. Mathers B, Degenhardt L, Phillips B, Wiessing L, Hickman M, Strathdee S, Wodak A, Panda S, Tyndall M, Toufik A. Global epidemiology of injecting drug use and HIV among people who inject drugs: a systematic review. The Lancet. 2008;372(9651):1733-1745.

7. Rossi D. VIH en personas que usan drogas en América Latina y el Caribe. En: Retos planteados por la epidemia del VIH en América Latina y el Caribe, 2009 [Internet]. Lima: ONUSIDA, OPS, UNICEF; 2009 [citado 12 may 2012] p. 31-42. Disponible en: www.unaids.org.br/biblioteca/ links/OPAS-OPS/OPAS\%2024.pdf.
8. Caiaffa WT, Zocratto KF, Osimani ML, Martínez PL, Radulich G, Latorre L, Muzzio E, Segura M, Chiparelli H, Russi J, Rey J, Vazquez E, Cuchi P, Sosa-Estani S, Rossi D, Weissenbacher $M$. Hepatitis $C$ virus among non-injecting cocaine users (NICUs) in South America: can injectors be a bridge? Addiction. 2011;106(1):143-151.

9. Muzzio E, Rajput S, Naveira J, Segura M, Vila M, Ralón G, Galante A, Drago SM, Bouzas MB, Zapiola I, Griemberg G, Deluchi G, Menga G, Cuchi P, Tang A, Weissenbacher M, Rossi D. Infecciones y otros problemas de salud en usuarios de pasta base de cocaína en un centro asistencial de Buenos Aires, Argentina. Revista Argentina de Salud Pública. 2010;1(4):14-19.

10. Osimani ML, Latorre L, Garibotto G, Scarlatta L, Chipparelli H, Vidal J. VIH, Hepatitis B, Hepatitis C y VDRL en usuarios de cocaína no inyectable en Uruguay. Adicciones. 2005; 17(2):157-162.

11. Pechansky F, von Diemen L, Kessler F, Hirakata V, Metzger D, Woody GE. Preliminary estimates of human immunodeficiency virus prevalence and incidence among cocaine abusers of Porto Alegre, Brazil. Journal of Urban Health. 2003;80(1):115-126.

12. Ralón G, Pando MA, Fassano A, Naveira J, Parga J, De Lisa R, Barros S, Verón D, Rossi D, Salomón H, Vila M. Seroincidencia y seroprevalencia de VIH en usuarios de drogas en Argentina. En: Actualizaciones en sida. Salta: Sociedad Argentina Interdisciplinaria de Sida, Fundación Huesped; 2009. p. 16.

13. Rossi D, Radulich G, Muzzio E, Naveira J, Sosa-Estani S, Rey J, Griemberg G, Friedman SR, Martínez-Peralta L, Weissenbacher M. Multiple infections and associated risk factors among noninjecting cocaine users in Argentina. Cadernos de Saúde Pública. 2008;24(5):965-974.

14. Zocratto KBF, Osimani $M L$, Peralta $L M$, Latorre L, Muzzio E, Segura M, Chiparelli H, Proietti FA, Rey J, Vazquez E, Cuchi P, Estani SS, Rossi D, Weissenbacher M, Caiaffa WT. Sexual behavior and HBV infection among noninjecting 
cocaine users (NICUs). Substance Use \& Misuse. 2010;45(12):2026-2044.

15. Malta M, Bastos FI, Silva CM, Pereira GFM, Lucena FF, Fonseca MG, Strathdee SA. Differential survival benefit of universal HAART access in Brazil: A nation-wide comparison of injecting drug users versus men who have sex with men. Journal of Acquired Immune Deficiency Syndromes. 2009;52(5):629-635.

16. Zala C, Rustad CA, Chan K, Khan NI, Beltran M, Warley E, Ceriotto M, Druyts EF, Hogg RS, Montaner J, Cahn P. Determinants of treatment access in a population-based cohort of HIV-positive men and women living in Argentina. Medscape Journal of Medicine [Internet]. 2008 [citado 12 may 2012];10(4):78. Disponible en: http://www.ncbi.nlm.nih.gov/pmc/articles/PMC2 390686/.

17. Blettner M, Sauerbrei W, Schlehofer B, Scheuchenpflug T, Friedenreich C. Traditional reviews, meta-analyses and pooled analyses in epidemiology. International Journal of Epidemiology. 1999;28(1):1-9.

18. Curran PJ, Hussong AM. Integrative data analysis: The simultaneous analysis of multiple data sets. Psychological Methods. 2009;14(2):81-100.

19. Friedenreich CM. Methods for pooled analyses of epidemiologic studies. Epidemiology. 1993;4(4):295-302.

20. Friedenreich CM. Commentary: Improving pooled analyses in epidemiology. International Journal of Epidemiology. 2002;31(1):86-87.

21. Samaja J. Epistemología y metodología: Elementos para una teoría de la investigación científica. 3ra ed. Buenos Aires: Eudeba; 1999.

22. Galtung J. Teoría y métodos de la investigación social. Buenos Aires: Eudeba; 1966.

23. Lazarsfeld P, Boudon, R. Metodología de las ciencias sociales: conceptos e índices (Tomo 1). Barcelona: Laia; 1973.

24. Derrida J. La estructura, el signo y el juego en el discurso de las ciencias humanas. En: La estructura y la diferencia. Barcelona: Antrophos; 1989. p. 383-401.

25. Díaz E. La construcción de una metodología ampliada. Salud Colectiva. 2010;6(3):263-274.

26. Caiaffa WT. Projeto AJUDE-Brasil: avaliação epidemiológica dos usuários de drogas injetáveis dos Projetos de Redução de Danos (PRD) apoiados pela CN-DST/Aids [Internet]. Brasília: Ministério da Saúde; 2001 [citado 12 may 2012]. http://bvsms.saude.gov.br/bvs/publicacoes/51 pro jeto_ajudeBrasil.pdf.

27. Caiaffa WT, Bastos FI, Freitas LL de, Mingoti SA, Proietti FA, Carneiro-Proietti AB, Gandolfi D, Doneda D. The contribution of two Brazilian multi-center studies to the assessment of HIV and HCV infection and prevention strategies among injecting drug users: the AJUDEBrasil I and II Projects. Cadernos de Saúde Pública. 2006;22(4):771-782.

28. Touzé G, Rossi D, Cymerman P, Ereñú N, Faraone S, Goltzman P, Rojas EJ, Vázquez S. Prevención del VIH/SIDA en usuarios de drogas: resultados de un proyecto de investigación e intervención. Buenos Aires: Intercambios Asociación Civil; 1999.

29. Hacker MA, Friedman SR, Telles PR, Teixeira $\mathrm{SL}$, Bongertz V, Morgado MG, Bastos FI. The role of "long-term" and "new" injectors in a declining HIV/AIDS epidemic in Rio de Janeiro, Brazil. Substance Use \& Misuse. 2005;40(1):99-123.

30. Hacker MA, Leite IC, Renton A, Torres TG de, Gracie R, Bastos FI. Reconstructing the AIDS epidemic among injection drug users in Brazil. Cadernos de Saúde Pública. 2006;22(4):751-760.

31. Touzé G, Vila M, Pawlowicz MP, Rossi D. Saberes y prácticas sobre drogas: El caso de la pasta base de cocaína. Buenos Aires: Intercambios Asociación Civil, Federación Internacional de Universidades Católicas; 2006.

32. Rossi D, editor. Sida y Drogas: Reducción de daños en el Cono Sur. Buenos Aires: Ministerio de Salud de la Nación, Intercambios Asociación Civil; 2001.

33. Caiaffa WT, Bastos FI, Proietti FA, Reis ÂCM, Mingoti SA, Gandolfi D, Doneda D. Practices surrounding syringe acquisition and disposal: effects of Syringe Exchange Programmes from different Brazilian regions: the AJUDE-Brasil II Project. International Journal of Drug Policy. 2003;14(5-6):365-371.

34. Rossi D, Pawlowicz MP, Cymerman P, Goltzman P, Touzé G. Sexual risk behaviour in cocaine users from Buenos Aires City [Internet]. 15th International Conference on the Reduction of Drug-Related Harm: Conference handbook. Melbourne, Australia: Australian Drug Foundation, International Harm Reduction Association, Centre for Harm Reduction; 2004. 
p. 136-137 [citado 12 may 2012]. Disponible en: http://www.ihra.net/contents/194 .

35. Rossi D, Pawlowicz MP, Cymerman $P$, Goltzman P, Touzé G, Nkowane M, Vázquez E, García Hamilton L, Weissenbacher M, Saxena S. Rapid assessment and response on sexual risk behavior in cocaine users from Buenos Aires City [Internet]. The XV International AIDS Conference Abstract Book. Bangkok: International AIDS Society; 2004 [citado 12 may 2012]. Disponible en: http://www.iasociety.org/Abstracts/A2173835.aspx.

36. Rossi D, Rangugni V. Cambios en el uso inyectable de drogas en Buenos Aires (19982003). Buenos Aires: Intercambios Asociación Civil, Ministerio de Salud y Ambiente de la Nación, ONUSIDA, ONUDD; 2004.

37. Osimani ML, Vázquez Pedrouzo $R$, Chipparelli H, Guchin M, Latorre L, Garibotto G, Gherardi Pérez A, Vidal J. Seroprevalencia para los virus de la inmunodeficiencia humana, hepatitis B y C en usuarios de drogas inyectables. Revista Médica del Uruguay. 2005;21(3):207214.

38. Rossi D, Pawlowicz MP, Rangugni V, Zunino Singh D, Goltzman P, Cymerman P, Vila M, Touzé G. The HIV/AIDS epidemic and changes in injecting drug use in Buenos Aires, Argentina. Cadernos de Saúde Pública. 2006;22(4):741-750.

39. Rossi D, Zunino-Singh D, Pawlowicz MP, Touzé G, Bolyard M, Mateu-Gelabert P, Sandoval M, Friedman SR. Changes in time-use and drug use by young adults in poor neighbourhoods of Greater Buenos Aires, Argentina, after the political transitions of 2001-2002: Results of a survey. Harm Reduction Journal [Internet]. 2011 [citado 12 may 2012];8(2). Disponible en: http://www.ncbi.nlm.nih.gov/pmc/articles/PMC3 039540/.

40. Braun V, Clarke V. Using thematic analysis in psychology. Qualitative Research in Psychology. 2006;3(2):77-101.

41. Thomas J, Harden A. Methods for the thematic synthesis of qualitative research in systematic reviews. BMC Medical Research Methodology [Internet]. 2008 [citado 12 may 2012];8(45). Disponible en: http://www.biomedcentral.com/ 1471-2288/8/45.

42. Morgado MG, Bastos Fl. Estimates of HIV-1 incidence based on serological methods: a brief methodological review. Cadernos de Saúde Pública. 2011;27(Suppl 1):S7-S18.
43. Brandt C, Cohen D, Shifman M, Miller P, Nadkarni P, Frawley S. Approaches and informatics tools to assist in the integration of similar clinical research questionnaires. Methods of Information in Medicine. 2004;43(2):156-162.

44. Martin ET, Krantz E, Gottlieb SL, Magaret AS, Langenberg A, Stanberry L, Kamb M, Wald A. A pooled analysis of the effect of condoms in preventing HSV-2 acquisition. Archives of Internal Medicine. 2009;169(13):1233-1240.

45. Egger M, Smith GD. Meta-analysis bias in location and selection of studies. British Medical Journal. 1998;316(7124):61-66.

46. Molinero-Casares LM. Metaanálisis: claves para interpretar una herramienta de investigación controvertida. Hipertensión. 2001;18(5):232240 .

47. Ruano-Ravina A, Figueiras A, Barros-Dios JM. El metaanálisis a debate. Medicina Clínica. 2002;119(11):439.

48. Glass GV. Primary, secondary and MetaAnalysis of research. Educational Research. 1976;10(5):3-8.

49. Glass GV. Meta-Analysis at 25. Special Education Programs Research Project Directors' Conference (1999 Jul 15) [Internet]. Washington DC: US Department of Education; 2000 [citado 12 may 2012]. Disponible en: http://www.gvglass. info/papers/meta25.html.

50. Barreto ML, Teixeira MG, Bastos FI, Ximenes RA, Barata RB, Rodrigues LC. Successes and failures in the control of infectious diseases in Brazil: social and environmental context, policies, interventions, and research needs. The Lancet. 2011;377(9780):1877-1889.

51. Pinto MT, Rodrigues ES, Malta TM, Azevedo R, Takayanagui OM, Valente VB, Ubiali EMA, Covas DT, Kashima S. HTLV-1/2 seroprevalence and coinfection rate in Brazilian first-time blood donors: an 11-year follow-up. Revista do Instituto de Medicina Tropical de São Paulo. 2012;54(3):123-130.

52. Dirección de sida y ETS. Boletín sobre el VIHsida en la Argentina. 2011;XIV(28).

53. Dirección General de Salud. Boletín epidemiológico. Situación epidemiológica del VIHSIDA en Uruguay (1983-2009). Montevideo: Ministerio de Salud Pública, DEVISAPPITS/SIDA; 2009. 
54. Souto FJD, Fontes CJF, Pignati LT, Pagliarini MES, Menezes VM, Martinelli ALC, Figueiredo JFC, Donadi EA, Passos AD. Risk factors for hepatitis C virus infection in Inland Brazil: an analysis of pooled epidemiological sectional studies. Journal of Medical Virology. 2012;84(5):756-762.

55. Bravo MJ, Mejía IE, Barrio G. Encuestas de comportamiento en CODAR: Herramientas básicas para vigilancia de segunda generación de la transmisión de VIH y otras ITSS en Consumidores de Drogas con Alto Riesgo [Internet]. Washington DC: Organización Panamericana de la Salud; 2008 [citado 12 may 2012]. Disponible en: http://www.paho.org/Spanish/AD/FCH/AI/VIH_d rogas.htm\#Encuestas.

\section{FORMA DE CITAR}

Ralón G, Rossi D, Vila M, Latorre L, Bastos FI, Caiaffa WT. De los estudios locales a una perspectiva regional: análisis integrado de datos secundarios en un proyecto colaborativo sobre vulnerabilidades asociadas al uso de drogas en Argentina, Brasil y Uruguay (1998-2004). Salud Colectiva. 2012;8(3):229-246.

Recibido el 29 de febrero de 2012

Versión final presentada el 27 de julio de 2012

Aprobado el 9 de agosto de 2012 